\title{
Research on dynamic evaluation method for smart grids in consideration of differentiation
}

\author{
Wei Zhang ${ }^{\mathrm{a}}$, Bo Hu${ }^{\mathrm{a}}$, Lin Liu ${ }^{\mathrm{a}}$, Han Huang ${ }^{\mathrm{a}}$, Zhinan Kan ${ }^{\mathrm{b}}$, Xiaobao Yu ${ }^{\mathrm{b}}$ \\ ${ }^{a}$ State Grid Energy Research Institute Beijing,102200 P.R.CHINA \\ ${ }^{b}$ North China Electric Power University Beijing, 102206 P.R.CHINA
}

\begin{abstract}
Reasonable evaluation on current conditions of smart grid is of vital practical significance to scientific instructions for smart grid planning and construction. Conventional evaluation method for smart grid is mainly static evaluation, which is of single evaluation standard, and without consideration of differentiation factors, which makes the evaluation results not objective and no comparable. A dynamic evaluation method for smart grid, which considers both indicator development differences and regional differences, is proposed in this paper. The method acquires comprehensive evaluation results from two aspects, including to modify evaluation values of properties based on analysis on differentiation degree of each indicator development and to modify weights of properties based on analysis on differentiation of regional development for smart grid. Data of three regions in the latest five years are selected for case study in the paper. Results indicate that compared with traditional methods without consideration of the difference, our method is more objective and reasonable, and is able to effectively find out problems of unbalanced development of power grid.
\end{abstract}

Keywords: Dynamic evaluation, smart grids, regional difference, indicator developmental difference

\section{Introduction}

Smart grid refers to a complex and huge system project. Comprehensive and scientific evaluation on benefits of smart grid and timely discovery of weak links and restraints in the development are of great practical significance to correct instructions for planning, construction, operation and management of smart grid. In the field of smart grid comprehensive evaluation, many research institutes have developed relevant studies. IBM has proposed the Smart Grid Maturity Model, dividing development of smart grid into 5 phases, which is applicable to instructing power enterprises on investment of smart grid [1]. The United States Department of Energy (DOE) presented the Smart Grid Evaluation System, fully demonstrating the understanding of American government on smart grid, highlighting the necessity of smart grid to provide users with better services and enhancing operation earnings of power system through user participation [2], [3]. The Smart Grid Benefits Assessment system, proposed by EU, was designed to boost the research and application of smart grid technology and equipment, and effective utilization of clean energy, to realize the target of energy conservation and emission reduction in EU [4]. Domestically, some indicator system were proposed, such as "resource-saving and environment-friendly" smart grid indicator system and smart grid development indicator system, making evaluations on the development of smart grid from the perspective of smart grid service on "resource-saving and environment-friendly" society and smart grid itself in respect to safety, economics, intelligence, etc. respectively [5]-[8].

In other fields, there are numerous studies on comprehensive evaluation methods [9], [10]. Yi PT et al.

\footnotetext{
* Manuscript received July 10, 2015; revised August 4, 2015.

Corresponding author. Tel.:+86-10-66603749; E-mail address: hubo@ sgeri.sgcc.com.cn

doi: $10.12720 /$ sgce.4.3.177-185
} 
[11] researched a multi-indicator dynamic comprehensive evaluation modification method for vertical and horizontal modification on multiple indicators by virtue of correction factors and modified functions targeted at evaluation on multi-indicator dynamic reward or punishment; Zhang HQ et al. [12] discussed basic concepts and applicable steps for common multi-indicator comprehensive evaluation method and made comparison on their advantages, disadvantages and applicable conditions, offering reasonable options for research on its application. Some scholars applied multi-indicator evaluation to evaluation on energy efficiency, adopting the Analytical Hierarchy Process (AHP) and three-dimensional structure model to make analysis on indicator evaluation; besides, other scholars used other methodologies to study multi-indicator comprehensive evaluation.

However, there are no studies made on multi-indicator and dynamic evaluation [13]. Most scholars only made analysis from the perspective of single indicator, lacking systematic researches. Moreover, studies on evaluation on construction of smart grid were generally static evaluation. The evaluation standard was lack of variety, resulting in the evaluation results being less scientific and comparable. In the paper, a dynamic evaluation method based on comprehensive evaluation indicator system and considered indicator development difference and regional difference is proposed.

Primarily, the second-level comprehensive evaluation indicator system for smart grid is designed, and the initial weight for indicator system is acquired by the AHP. Next, Differentiated Impact Factor (DIF) is calculated through analysis on differentiation development of second-level indicators, to modify the values of the second-level indicators. Then, weights of first-level indicators are modified by analysis on regional difference. Finally, based on modified evaluation values and weights for indicators, a dynamic evaluation is made on smart grid. A case will be studied to prove the validity of the method.

Table 1. Comprehensive evaluation indicator system for smart grid

\begin{tabular}{|c|c|c|}
\hline $\begin{array}{c}\text { Evaluation } \\
\text { Indicator System }\end{array}$ & $\begin{array}{l}\text { First-level Indicator } \\
\text { (property) }\end{array}$ & Second-level Indicator \\
\hline \multirow{19}{*}{$\begin{array}{l}\text { Comprehensive } \\
\text { Evaluation } \\
\text { Indicator System } \\
\text { (U) }\end{array}$} & \multirow{4}{*}{ Strongness (A1) } & Gross installed capacity (B11) \\
\hline & & $\mathrm{N}-1$ pass rate $(\mathrm{B} 12)$ \\
\hline & & Capacity-load ratio (B13) \\
\hline & & Intelligent substation proportion (B14) \\
\hline & \multirow{3}{*}{ Reliability (A2) } & Voltage qualification rate (B21) \\
\hline & & Power supply reliability (B22) \\
\hline & & Customer average interruption time (B23) \\
\hline & \multirow{3}{*}{ Efficiency (A3) } & Power grid transmission losses (B31) \\
\hline & & Accuracy for smart diagnosis (B32) \\
\hline & & Organization GDP power consumption (B33) \\
\hline & \multirow{3}{*}{ Economy (A4) } & Electricity sales for organization power grid assets (B41) \\
\hline & & Power grid dissipation discount (B42) \\
\hline & & Electricity sales increase for organization power grid investment (B43) \\
\hline & \multirow{3}{*}{ Environmental Friendly (A5) } & Clean energy capacity (B51) \\
\hline & & Carbon dioxide reduction (B52) \\
\hline & & Environmental impact assessment (EIA) pass rate (B53) \\
\hline & \multirow{3}{*}{ Interaction (A6) } & Proportion of power customer service system (B61) \\
\hline & & Customer satisfaction (B62) \\
\hline & & Proportion of smart electricity meters (B63) \\
\hline
\end{tabular}




\section{Comprehensive Evaluation Indicator System for Smart Grid}

Building appropriate evaluation indicator system for smart grid is the premise for comprehensive evaluation. Indicator system shall fully cover all links of smart grid, highlight new technology and focus for implementation in smart grid and overall reflect the difference between smart grid and traditional power grid. Besides, indicators shall be specifically defined and measurable. Some key indicators are selected from six aspects including strongness, reliability, efficiency, economics, environment and interaction to build the indicator system for the comprehensive evaluation based on existing evaluation indicator systems for smart grid in the paper [14]. Indicator system is composed of 6 first-level indicators (properties) and 19 second-level indicators, as shown in Table 1.

\section{Differentiated Analysis on Smart Grid Evaluation}

The regional differences in the development of smart grid reflect different features in different time, spatial levels and spatial patterns, and the focus, time sequence and scale in the development of smart grid are also different. In the evaluation on smart grid, difference is an important factor required for consideration. In the paper, two factors are generally taken into consideration: indicators developmental difference and regional difference.

\subsection{Indicators developmental difference}

In previous comprehensive evaluations, evaluation weight was basically static and comprehensive evaluation value was equal to weight of all indicators, which was likely to result in less focus on development of indicators with smaller weight due to too much emphasis on development of indicators with larger weight. In the meantime, since relevance exists among indicators, abnormal construction of smart grid is bound to occur if neglecting such a relevance and blindly developing several indicators, which is not conducive to the comprehensive and healthy development of smart grid. It is necessary to take difference in the development of indicators into account and incorporate it as an impact factor into dynamic evaluation on the development of smart grid when formulating evaluation weight.

\subsection{Regional difference}

Since different regions are different in natural resources, population density, economic growth etc., different regions focus differently in the construction of smart grid. In regions where there are abundance of natural resources, there are more power supply points with larger capacity, which is of superiority compared with other regions in terms of strongness; in developed regions, more investment has been made on power grid with more advanced equipment, which is advantageous in terms of economics and reliability. If utilizing the same set of weight to make evaluation on smart grid in all the regions, it shall be difficult to give same consideration to regional difference. Therefore, when making evaluation on smart grid in different regions, it shall be necessary to incorporate the difference as an impact factor into evaluation.

\section{Dynamic Evolution Method}

Computational flow for differentiated evaluation method is shown in Fig. 1. The evaluation is generally divided to four steps.

1) Initial processing on indicator weight. Base on grades given by experts according to the importance of indicators, applying the AHP to obtain the initial weights for first-level indicators and second-level indicators, as shown in Fig. 2.

2) Modify value of first-level indicators (properties). Make dimensionless treatment on second-level indicators and calculate the Indicator Synergy Development Coefficient (ISDC) so as to get DIF for indicators developmental difference to modify value of first-level indicators (properties).

3) Modify weights for first-level indicators. Base on regional difference, calculate the judgment matrix and Property Weight Correction Matrix (PWCM) to modify initial weights for first-level indicators and obtain modified first-level indicator weights. 
4) Determine dynamic evaluation value. Base on modified value and weights of first-level indicators to acquire the dynamic evaluation result.



Fig. 1. Flow chart for dynamic evaluation on smart grid.

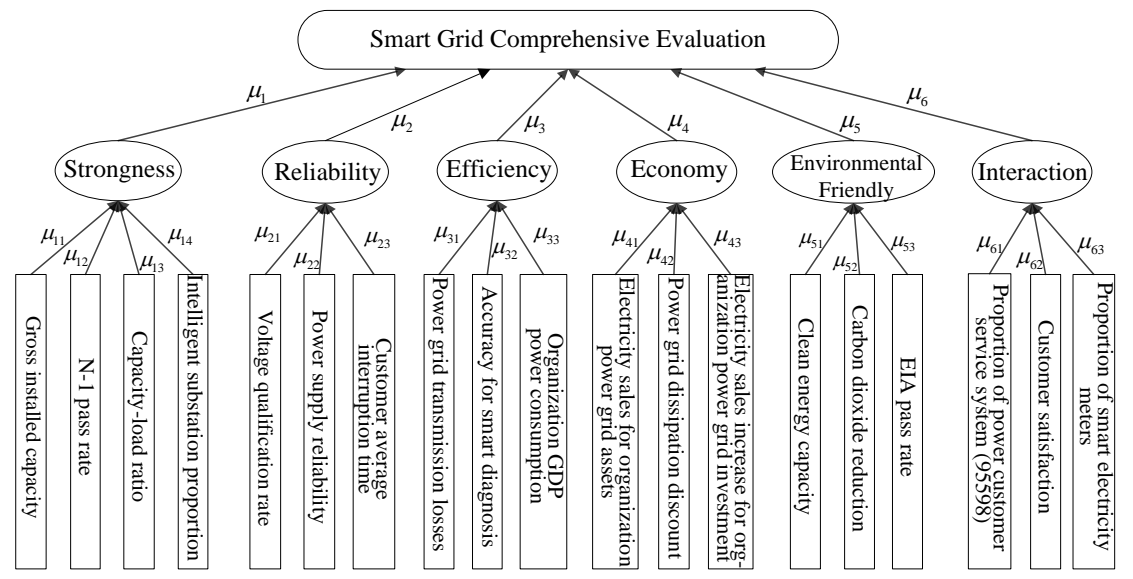

Fig. 2. Structural chart of comprehensive evaluation indicator system.

\subsection{Indicators developmental difference}

Indicators developmental difference is based on actual data of second-level indicators, and determine differentiated impact factor by analyzing ISDC for each property to modify the property value. Assume that there are $\mathrm{M}$ evaluation objects and $\mathrm{N}$ indicators in each property. The procedure of the algorithm is as follows:

Step 1) Data collection. Collect indicator data of the evaluation object of the latest $\mathrm{T}$ years, which are recorded as $x_{i j k}$ to indicate the actual value of $k$ indicators of region $i$ in year $j$.

Step 2) Dimensionless treatment. Maximum-Minimum method is used to make dimensionless treatment on all the data. The equation is

$$
y_{i j k}=\frac{x_{i j k}-\min _{i=1, j=1}^{M, T} x_{i j k}}{\max _{i=1, j=1}^{M, T} x_{i j k}-\min _{i=1, j=1} x_{i j k}} \in[0,1]
$$


Step 3) Calculate of ISDC. Calculate ISDC for the region. Take Indicator 1 (B11) and Indicator 2 (B12) as an example. The calculation equation is

$$
r_{12}=\frac{\sum_{j=1}^{T} y_{i j 1} y_{i j 2}-\frac{\sum_{j=1}^{T} y_{i j 1} \sum_{j=1}^{T} y_{i j 2}}{T}}{\sqrt{\left(\sum_{j=1}^{T} y_{i j 1}^{2}-\frac{\left(\sum_{j=1}^{T} y_{i j 1}\right)^{2}}{T}\right)\left(\sum_{j=1}^{T} y_{i j 2}^{2}-\frac{\left.\left(\sum_{j=1}^{T} y_{i j 2}\right)^{2}\right)}{T}\right)}}
$$

Step 4) Calculate of DIF. It is known through calculation that there are $C_{n}^{2}$ correlation coefficients among $n$ indicators. Select an absolute value of any two calculation differentials and then get to that $\phi_{p}, p=1,2, \ldots, C_{n}^{2}$. Calculation principle for DIF is

$$
\begin{aligned}
& \phi_{p}=\sum_{s_{1}=1, s_{2}=2}^{C_{n}^{2}, C_{n}^{2}}\left|r_{s_{1} p}-r_{s_{2} p}\right|, s_{1} \neq s_{2} \\
& \theta_{i}=1-\frac{\sum_{p=1}^{C_{n}^{2}} \phi_{p}}{C_{n}^{2}} \in[0,1]
\end{aligned}
$$

where, $\theta_{i}$ is the DIF for the region $i$. When calculating the evaluation value for the property, use it to modify the evaluation value. Take the property $q$ as an example. The equation of evaluation value for property $q$ of region $i$ in the year $j$ is

$$
\mathrm{A}_{i j q}=\left(\sum_{k=1}^{n} \mu_{q k} \bullet y_{i j k}\right) \times \theta_{i q}
$$

where, $\mu_{q k}$ is initial weight for the indicator $k$ of property $q$.

\subsection{Regional difference}

There are different construction focus, time sequence and scale for each region. In another word, the property is of different importance degrees in different regions, which can be gain by the expert scoring method. According to the importance degree of the property in different regions, modify weights of properties.

Use $\alpha_{i_{1} i_{2}}$ to signify the comparison value of important degree between Region $i_{1}$ and Region $i_{2}$, when $i_{1}=i_{2}, \alpha_{i_{1} i_{2}}=1$. The comparison rules are shown in Table 2 .

Table 2. Comparison rule for properties

\begin{tabular}{|c|c|c|c|c|c|}
\hline & $\begin{array}{c}\text { Equally } \\
\text { important }\end{array}$ & $\begin{array}{c}\text { Slightly } \\
\text { important }\end{array}$ & $\begin{array}{c}\text { Relatively } \\
\text { important }\end{array}$ & $\begin{array}{c}\text { Very } \\
\text { important }\end{array}$ & $\begin{array}{c}\text { Absolutely } \\
\text { important }\end{array}$ \\
\hline$\alpha_{i_{1} i_{2}}$ & 1 & 3 & 5 & 7 & 9 \\
\hline
\end{tabular}


According to the symmetry principle, $\alpha_{i_{2} i_{1}}=1 / \alpha_{i_{1} i_{2}}$.

The procedure of regional difference calculation is as follows.

Step 1) Calculate judgment matrix. According to the comparison rules, the judgment matrix is calculated as follows:

$$
\alpha \rightarrow \beta=\left[\begin{array}{cccc}
\alpha_{11} & \alpha_{12} & \ldots & \alpha_{1 n} \\
\alpha_{21} & \alpha_{22} & \ldots & \alpha_{2 n} \\
\ldots & \ldots & \ldots & \ldots \\
\alpha_{n 1} & \alpha_{n 2} & \ldots & \alpha_{n n}
\end{array}\right]
$$

Step 2) Property Weight Correction Matrix. Calculate the root of the product of each line

$$
\xi_{i}=\sqrt[n]{\prod_{i_{2}=1}^{n} \alpha_{i_{1} i_{2}}}
$$

Carry out the normalization processing of the root to get the Property Weight Correction Matrix

$$
\beta_{i q}=\frac{\xi_{i}}{\sum \xi_{i}}
$$

Step 3) Modify the weights of property. According to Property Weight Correction Matrix, modify the weights of the properties

$$
\lambda_{q}=\frac{\mu_{q} \bullet \beta_{i q}}{\sum_{q=1}^{6} \mu_{q} \bullet \beta_{i q}}
$$

where, $\mu_{q}$ is the initial property weights without consideration of regional differences.

Step 4) Calculate dynamic evaluation value. Combine with the formula (5) and (9), calculate the dynamic evaluation value. The Evaluation for property $q$ of region $i$ in the year $j$ is

$$
\mathrm{B}_{i j}=\sum_{q=1}^{6} A_{i j q} \bullet \lambda_{q}
$$

\section{Case Study}

The data of three regions (R1, R2 and R3) in recent five years are collected. The conventional evaluation method and dynamic evaluation method are applied to evaluate the three regions respectively, to demonstrate the validity of our method.

Table 3. Initial indicator value and weights of property A2

\begin{tabular}{|l|l|l|l|l|l|}
\hline A2 & B21 & B22 & B23 & weight & Consistency test \\
\cline { 1 - 4 } B21 & 1.00 & 1.67 & 2.33 & 0.47 & $\lambda_{\max }=3.0829$ \\
B22 & 0.67 & 1.00 & 2.33 & 0.35 & $\begin{array}{l}\text { C }=041459 \\
\text { CR }=0.071481\end{array}$ \\
\hline B23 & 0.44 & 0.44 & 1.00 & 0.18 & \\
\hline
\end{tabular}




\subsection{Initial weights setting}

Firstly, calculate the initial weights of each indicator by AHP and the degree of importance for indicators commented by experts. Take the property A2 (Reliability) as an example shown in Table 3.

Remark 1: According to the expert advice, if the evaluation value is higher than 0.5 (Standard Value), the region could be deemed in good state of development.

\subsection{Conventional evaluation method}

Firstly, the evaluation method without consideration of differences was used to analyze the development situation for three regions in 2013 based on the five-year data. The result is shown in Table 4.

Table 4. Conventional evaluation results for three regions

\begin{tabular}{|l|l|l|l|l|l|l|l|}
\hline $\mathbf{2 0 1 3}$ & $\mathbf{A 1}$ & $\mathbf{A 2}$ & $\mathbf{A 3}$ & $\mathbf{A 4}$ & $\mathbf{A 5}$ & $\mathbf{A 6}$ & Evaluation \\
\hline R1 & 0.71 & 0.78 & 0.71 & 0.38 & 0.96 & 0.45 & 0.691 \\
\hline R2 & 0.67 & 0.77 & 0.31 & 0.63 & 0.92 & 0.92 & 0.663 \\
\hline R3 & 0.70 & 0.83 & 0.29 & 0.34 & 0.94 & 0.80 & 0.637 \\
\hline
\end{tabular}

Based on the results above, the comprehensive evaluation value for three regions exceed the standard value (0.5), which means all three regions enjoy good development of smart grid.

\subsection{Dynamic evaluation method}

According to the calculation principle of indicators developmental difference and regional difference, dynamic evaluation analysis is conducted on the three regions in 2013. The results are shown in Table 5.

Table 5. Dynamic evaluation result for three regions

\begin{tabular}{|l|l|l|l|l|l|l|l|}
\hline $\mathbf{2 0 1 3}$ & $\mathbf{A 1}$ & $\mathbf{A 2}$ & $\mathbf{A 3}$ & $\mathbf{A 4}$ & $\mathbf{A 5}$ & A6 & Evaluation \\
\hline R1 & 0.68 & 0.76 & 0.51 & 0.35 & 0.96 & 0.39 & 0.652 \\
\hline R2 & 0.68 & 0.74 & 0.22 & 0.62 & 0.88 & 0.80 & 0.502 \\
\hline R3 & 0.36 & 0.47 & 0.28 & 0.28 & 0.87 & 0.79 & 0.412 \\
\hline
\end{tabular}

As seen from the results above, the comprehensive evaluation value was 0.65 for R1 in 2013, 0.5 for R2 and 0.41 for R3. According to comprehensive evaluation rule mentioned above, only R1 was well above the standard, while R2 was just reaching the standard and R3 falling below 0.5. In other words, the development of smart grid in R3 is problematic.

Furthermore, it is could be found that not all the indicators' scores in R3 are lower than standard value. In particular, the R3 was in a relative advantage in the properties of environment and interaction, despite a negative evaluation of economics, which suggests the R3's smart grid overemphasis on the importance of environment with insufficient attention to economics. Such unbalanced development is not conducive to the future development of smart grid. By comparison, R1 enjoyed a coordinated sustainable development of all indicators, in spite of no outstanding development on certain indicators. Therefore, the ISDC is large, which contributed to the evaluation value and all positive outcomes.

\subsection{Comparative analysis of the evaluation results}

According to the calculation procedure of 2013, the non-differentiated comprehensive evaluation values and differentiated comprehensive evaluation values of 2009 to 2012 can also be calculated, the result shown in Table 6 and the historical tendency of evaluation values shown in Fig. 3. 
Table 6. Evaluation results of two methods from 2009 to 2013

\begin{tabular}{|l|l|l|l|l|l|l|}
\hline \multirow{2}{*}{ Years } & \multicolumn{2}{l}{ R1 } & \multicolumn{2}{l}{ R2 } & \multicolumn{2}{l|}{ R3 } \\
\cline { 2 - 7 } & CON & DYN & CON & DYN & CON & DYN \\
\hline 2009 & 0.308 & 0.230 & 0.408 & 0.367 & 0.542 & 0.351 \\
\hline 2010 & 0.449 & 0.361 & 0.566 & 0.551 & 0.589 & 0.381 \\
\hline 2011 & 0.533 & 0.456 & 0.575 & 0.495 & 0.649 & 0.418 \\
\hline 2012 & 0.558 & 0.543 & 0.570 & 0.389 & 0.608 & 0.402 \\
\hline 2013 & 0.691 & 0.652 & 0.663 & 0.502 & 0.637 & 0.412 \\
\hline
\end{tabular}

Remark: CON->conventional evaluation results, DYN->dynamic evaluation results.

As seen from historical evaluation results, R3 shows the highest comprehensive evaluation value without the consideration the differences. Nevertheless, the value drops substantially after the introduction of the two differences, making R3 lag behind the other two regions. As discovered from the analysis of the indicators of R3, the substantial drop is caused by seriously biased smart grid construction with unbalanced indicator development, which is not beneficial to the future development of smart grid.

Comprehensive analysis of R3 leads to the finding that the former evaluation method without considering indicators development and regional differences is defective. On the other hand, the dynamic evaluation method proposed by this paper can address the defect and effectively check the unbalance development of smart grid due to chasing the higher evaluation. Based on the analysis results, policy suggestions can be offered to relevant regions for rationalized and healthy development of smart grid.

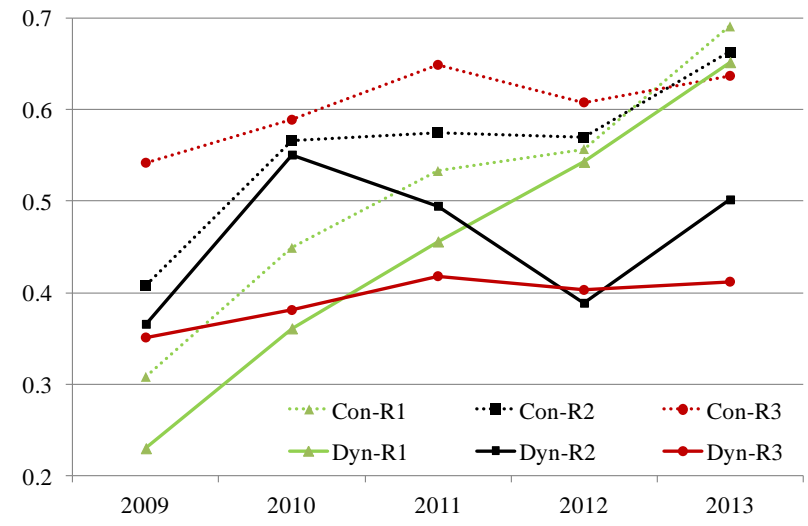

Fig. 3. Profiles of evaluation results of two methods.

\section{Conclusion}

This paper proposed a dynamic evaluation method for smart grid that accommodates both indicator developmental difference and regional difference. Firstly, based on the existing comprehensive evaluation indicator system for smart grid, a system composed of six first-level indicators (properties) and 19 secondlevel indicators was formed, covering six aspects, i.e. strongness, reliability, efficiency, economics, environment and interaction. Secondly, the secondly-level indicators developmental difference was analyzed to modify the evaluation value of each property, and the weight of each property was modified upon analysis of regional development of smart grid. Finally, the weighting of properties and weights determined the dynamic evaluation values.

Case-based comparative analysis proved the effectiveness of this method. First, the comprehensive evaluation values of smart grid of the three regions were calculated by conventional evaluation method without considering differentiation, showing positive results for all these three regions. Then, the method proposed in this paper was adopted for dynamically evaluating the smart grid of the three regions, leading 
differentiated evaluation values. In particular, R3 slipped from good to problematic state with unbalanced development of indicators, due to its strongly biased development of its smart grid. Through the case, it can be found that the dynamic evaluation method proposed by this paper can address the defect of "emphasis on indicators of large weight and less attention to those of small weights" in the conventional method. Besides, proposed method can effectively eliminate the problem, which is the unbalanced development of smart grid caused by the chase of higher evaluation score. Based on the analysis results, policy suggestions can be offered to relevant regions for rationalized and healthy development of smart grid.

\section{References}

[1] Software Engineering Institute of CMU. Smart grid maturity model-SGMM model definition. Pittsburgh: Carnegie Mellon University; 2011.

[2] U. S. DOE. Smart Grid Characteristics, Values, and Metrics. Washington: U.S. DOE; 2010.

[3] U. S. EPRI. Methodological Approach for Estimating the Benefits and Costs of Smart Grid Demonstration Projects. Palo Alto: U. S. EPRI; 2010.

[4] European Commission. European Technology Platform Smart Grids: Vision and Strategy for Europe's Electricity Networks of the Future. Brussels: European Commission; 2010.

[5] Wang ZD, Li H, Li J, et al. Assessment index system for smart grids. Power System Technology, 2009; 33(17):14-18.

[6] Yang F, Zhao SQ. Impact of smart grid development on composite power system reliability evaluation. Power System and Clean Energy, 2013; (7):24-30.

[7] Ding Q, Li LL, Tu MF, et al. Design and key technologies of day-ahead generation scheduling system for smart grid. Power System and Clean Energy, 2013; (9):1-5.

[8] Tan W, He GY, Liu F, et al. A preliminary investigation on smart grid's low-carbon index system. Automation of Electric Power Systems, 2010; 34(17):1-5.

[9] Liu HY, Hao H, Kong F. Improvement on sequential multi-index firm performance evaluation. Electric Power Science and Engineering, 2008; 4:35-37.

[10] Liu D, Chen XQ, He GY, Zhou SX. Supplier-customer interaction modeling in an integrated optimal spot pricing framework by nonlinear point methods. Automation of Electric Power Systems, 2005; 23(2):7-14.

[11] Yi PT, Zhaog D. Study on dimensionless methods in dynamic comprehensive evaluation. Journal of Northeastern University (Natural Science), 2009; 30(6):889-892.

[12] Zhang HQ, Zhao YL. Survey on the multi-index comprehensive evaluation method. Modern Business Trade Industry, 2014; 26(7):20.

[13] Zhao J, Wu Y, Zhu N. Check and evaluation system on heat metering and energy efficiency retrofit of existing residential buildings in northern heating areas of china based on multi-index comprehensive evaluation method. Eenrgy Policy. 2009; 37:2124-2130.

[14] Sun Q, Ge XB, Liu L, et al. Multi-attribute network process comprehensive evaluation method for smart grid and its application. Power System Technology, 2012; 36(10):49-54. 